\title{
Expertentreffen Obstruktive Atemwegserkrankungen: Targets und Methoden *
}

\author{
Meeting of Experts on Obstructive Airway Diseases: Targets and Methods
}

Autoren

Institute
J. Lorenz ${ }^{1}$, R. Bals ${ }^{2}$, H.-U. Kauczor ${ }^{3}$, M. Pfeifer ${ }^{4}$, W. Randerath ${ }^{5}$, G. Steinkamp ${ }^{6}$, C. Taube ${ }^{7}$, H. Teschler ${ }^{8}$, T. Welte ${ }^{9}$, H. Worth ${ }^{10}$

Die Institutsangaben sind am Ende des Beitrags gelistet.

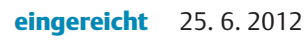
akzeptiert nach Revision 12. 7.2012

\section{Bibliografie}

DOI http://dx.doi.org/ 10.1055/s-0032-1310130 Pneumologie 2012; 66: 526-538 (c) Georg Thieme Verlag KG Stuttgart · New York ISSN 0934-8387

\section{Korrespondenzadresse} Prof. Dr. med. Joachim Lorenz Klinik für Pneumologie, Internistische Intensivmedizin, Infektiologie und Schlafmedizin Klinikum Lüdenscheid Paulmannshöher Straße 14 58515 Lüdenscheid Joachim.lorenz@klinikumluedenscheid.de

\section{Zusammenfassung \\ $\nabla$}

Zur Pathogenese und Klinik der chronisch obstruktiven Lungenerkrankung (COPD) und des Asthma bronchiale liegen zahlreiche neue Ergebnisse vor. Es wurden systematische Analysen von wohldefinierten und intensiv untersuchten Patientenkohorten publiziert, wie von der ECLIPSEKohorte in den USA und dem COSYCONet in Deutschland. Zentrale Publikationen des Jahres 2011 zur COPD und zum Asthma stellen bisherige Vorstellungen in Frage. Das pathogenetische Verhältnis von Parenchymdestruktion und bronchialer Obstruktion bei der COPD wird neu gesehen und die Bedeutung der kardiovaskulären Komorbidität neu bewertet. Die Bildgebung mit Computertomografie erlaubt die hochauflösende Darstellung der Lungenstruktur, und die MRT liefert zusätzlich funktionelle Informationen. Die Sicht der Patienten rückt stärker in den Fokus und wird mit Lebensqualitätsfragebögen, DyspnoeSkalen und dem COPD Assessment Test (CAT) dokumentiert. Das GOLD-Komitee unternimmt den Versuch, eine praktikable Klassifikation für die multiplen Facetten der COPD zu erarbeiten. Für die Therapie der COPD liegen Daten zu Betarezeptor-Antagonisten vor, ebenso wie zum Stellenwert der Anticholinergika für die Asthmatherapie. Diese Schwerpunkte waren Thema einer Expertentagung und werden im Übersichtsartikel zusammengefasst.

\section{Abstract \\ $\nabla$}

New insights into the pathogenesis and clinical course of chronic obstructive pulmonary disease (COPD) and asthma have become available. Systematic analyses of well-defined and intensively monitored patient cohorts are being published, particularly from the ECLIPSE cohort in the USA and from the network COSYCONet in Germany. Important articles from 2011 on COPD and asthma put former concepts into question. There is a new understanding of the relationship between parenchymal destruction and bronchial obstruction in COPD as well as on the impact of cardiovascular comorbidity. Computed tomography allows high-resolution imaging of lung structures, and MRI delivers supplementary functional information. Researchers have also investigated the value of patient-reported outcomes, such as quality of life, dyspnoea, or the COPD assessment test (CAT). Members of the GOLD committee are trying to establish a feasible classification of the multiple facets of COPD. With respect to treatment, novel data on beta-adrenergic antagonists in COPD and on muscarinic antagonists in asthma have been published. These aspects were discussed during an expert meeting and are now summarised in the present review article.

\footnotetext{
* Sponsor: Boehringer Ingelheim Pharma GmbH \& Co KG
} 
Year in review: COPD 2011

\section{$\nabla$}

\section{Helmut Teschler, Essen}

\section{Prävalenz der COPD im Vergleich zu} anderen chronischen Erkrankungen

Eine der wichtigen Publikationen zur COPD aus dem Jahre 2011 beschäftigt sich mit dem Risiko, im Alter zwischen 50 und 80 Jahren eine COPD zu entwickeln [1]. Dazu nutzten die Forscher eine longitudinale Kohorte von 13 Millionen Menschen aus der nationalen Gesundheitsdatenbank in Ontario, Kanada. Analysiert wurden Personen, die 1996 noch keine COPD hatten. 14 Jahre später wurde überprüft, wie viele Individuen eine COPD entwickelt hatten und 80 Jahre alt geworden oder verstorben waren. Unterschieden wurde zwischen der COPD-Diagnose in den originalen Fallbeschreibungen und der durch Spezialisten. Mehr als 500000 Individuen entwickelten im Beobachtungszeitraum eine COPD. Wendete man eine hochspezifische Falldefinition an, betrug das Lebensrisiko für COPD im Alter von 70 Jahren etwa $5 \%$ und mit 80 Jahren etwa $12 \%$. Die entsprechenden Werte unter Nutzung der allgemeineren COPD-Definition lagen mit 16\% bzw. 27\% deutlich höher. Die Autoren verglichen die COPD auch mit anderen chronischen Erkrankungen wie Herzinsuffizienz, Diabetes oder Asthma. Bei älteren Menschen war das COPD-Risiko deutlich höher als das für eine Herzinsuffizienz. Diese Daten zur Häufigkeit einer COPD im Alter dürften nicht nur für Kardiologen überraschend sein und unterstützen die Einschätzung der WHO.

Obstruktion kleiner Atemwege und Emphysem bei COPD Eine weitere sehr lesenswerte Arbeit beschäftigt sich mit der Frage, warum bei COPD die periphere Resistance erhöht ist [2]. Untersucht wurden Lungenpräparate von 78 Transplantat-Empfängern mit COPD sowie Präparate aus Spenderlungen. Die Gewebeproben wurden an definierten Stellen entnommen. Mit speziellen CT-Verfahren wurden Atemwege mit einem Durchmesser zwischen 2,0 und 2,5 mm identifiziert und das Ausmaß des Emphysems bestimmt. Die Zahl dieser terminalen Atemwege nahm mit höherem GOLD-Stadium progressiv ab. Pro Lungeneinheit gingen bei fortgeschrittener Erkrankung mehr kleinste Atemwege verloren: Im Stadium GOLD 0 fand man noch knapp 180, im GOLD-Stadium IV jedoch nur noch etwa 50 Bronchioli terminales pro Lungeneinheit. Besonders beim zentroazinären, aber auch beim panazinären Emphysem waren Gesamtzahl und Gesamtquerschnitt der kleinen Atemwege substanziell reduziert. Offenbar gingen eine Einengung und ein Verlust von terminalen Bronchiolen dem emphysematösen Umbau voraus. Dies würde im Einklang mit einer starken Erhöhung der peripheren Resistance stehen.

Das zugehörige Editorial geht auf die Frage ein, ob das Emphysem eher eine Erkrankung der kleinen Atemwege oder des Lungenparenchyms ist [3]. Für die erstere Hypothese sprechen Entzündungsprozesse, die zur Destruktion elastischer Fasern führen, die radiär im Bronchus liegen. Dies würde bedeuten, dass die Zerstörung der Alveolen ein sekundärer Prozess ist, der auf die Inflammation und Destruktion der kleinen Atemwege folgt.

\section{Symptomvariabilität bei COPD}

Die Variabilität der COPD-Symptomatik wurde in einer paneuropäischen Querschnittsstudie untersucht [4]. Aus 17 Ländern wurden 3642 Patienten mit schwerer oder sehr schwerer COPD mit dem Ziel untersucht, Schwankungen im Laufe des Tages zu identifizieren. Atemnot war mit $72 \%$ das häufigste Symptom. Die meisten Patienten berichteten über unterschiedlich starke Symptome im Verlaufe des Tages. Am stärksten waren Atemnot und Husten nach dem Aufwachen und am Morgen, während sie nachmittags und abends meist geringer ausgeprägt waren. Diese Daten unterstützen die morgendlichen „Anlaufschwierigkeiten“, über die viele COPD-Patienten in der Sprechstunde berichten. Der individuelle Therapieplan sollte dies berücksichtigen und beispielsweise die Physiotherapie eher nicht für den Morgen vorsehen.

\section{COPD-Exazerbation und kardialer Stress}

Die Interaktion zwischen Herz und Lunge ist auch bei COPD-Exazerbationen zu berücksichtigen. Hier besteht ein klarer Zusammenhang zwischen kardiovaskulärer Komorbidität und Letalität der COPD [5]. Wenn bei COPD-Exazerbation die Marker für kardiale Dysfunktion, NT-proBNP oder Troponin T, normal waren, lag die 30-Tage-Letalität nur bei etwa $2 \%$. Erhöhte Marker waren dagegen mit einer $28 \%$ igen Letalität verbunden. Ob diese Marker eine klinische Relevanz im Vergleich zu den Routine-Tests bieten, kann noch nicht abschließend beurteilt werden.

\section{Einflussfaktoren auf den FEV1-Verlauf}

Die ECLIPSE-Studie ist ein Projekt, bei dem mehr als 2000 COPDPatienten aus den Stadien Gold II bis IV über 36 Monate prospektiv verfolgt wurden [6]. Alle Patienten wurden von Spezialisten behandelt. Sie wurden verglichen mit 336 gesunden Rauchern und 246 gesunden Nichtrauchern. Bei allen Teilnehmern wurde der Abfall der FEV1 über die Zeit gemessen. Für die Gesamtpopulation betrug der Abfall an postbronchodilatatorischer FEV1 im Mittel $33 \mathrm{ml}$ pro Jahr. In den Gold-Stadien II, III und IV waren es durchschnittlich $35 \mathrm{ml}, 33 \mathrm{ml}$ und $25 \mathrm{ml}$ pro Jahr. Bemerkenswert war, dass man bei $15 \%$ der Patienten eine Verbesserung der FEV1 mit der Zeit feststellte. Ob es sich um einen echten Therapieeffekt oder um ein statistisches Phänomen handelt, ist noch offen. Überraschenderweise hatten Exazerbationen nur einen relativ geringen Einfluss auf den FEV1-Verlauf; im Mittel ergab sich pro Exazerbation ein um $2 \mathrm{ml}$ pro Jahr verstärkter Abfall der FEV1. Deutlich größere Auswirkungen auf einen beschleunigten FEV1Abfall hatte der Raucherstatus ( $-20 \mathrm{ml}$ FEV1 pro Jahr), ein Emphysem im CT $(-12 \mathrm{ml})$ und die Reversibilität nach Gabe eines Bronchodilatators $(-17 \mathrm{ml})$.

\section{Therapie mit Beta-Blockern und Überleben}

In einer retrospektiven Kohortenstudie aus Schottland wurde untersucht, welchen Einfluss die Anwendung von Beta-Blockern auf die COPD-Mortalität hat [7]. Bei den 5977 Patienten mit einem Durchschnittsalter von 69 Jahren betrug die durchschnittliche Beobachtungszeit gut vier Jahre. Insgesamt erhielten 819 Patienten eine additive Therapie mit Beta-Blockern, zu 88\% mit kardioselektiven Substanzen. Über die Indikation dieser Therapie und die Auswahl des Arzneimittels wird keine Aussage gemacht. Insgesamt 2005 Patienten verstarben während der Studie, entsprechend einer jährlichen Mortalität von $34 \%$. Patienten mit einer Beta-Blocker-Therapie hatten eine um $22 \%$ geringere Sterblichkeit. Diese Studie sollte Anlass sein, den Nutzen der Beta-Blocker-Therapie bei COPD in prospektiven Studien zu überprüfen.

\section{Roflumilast und Exazerbationen}

Der selektive Phosphodiesterase-Typ-4-Inhibitor Roflumilast ist zugelassen für Patienten mit schwerer oder sehr schwerer COPD, die häufig exazerbieren. Eine aktuelle Studie unterstützt diese Indikation [8]. Bei mehr als 3000 Patienten wurde Roflumilast oder 
Plazebo als Add-on zu einer Behandlung mit langwirksamen Betamimetika oder mit kurzwirksamen Muscarinantagonisten verabreicht. Mittelgradige und schwere Exazerbationen traten in der Gesamtgruppe unter Plazebo 1,4 mal pro Jahr auf, während sie unter Roflumilast mit 1,1 mal pro Jahr signifikant seltener waren. Patienten mit zwei oder mehr Exazerbationen profitierten deutlich von Roflumilast.

\section{Prävention von Exazerbationen mit Azithromycin}

In einer prospektiven, randomisierten klinischen Prüfung wurden 1100 Patienten mit COPD entweder täglich mit 250 mg Azithromycin oder mit Plazebo behandelt. [9]. Die Zahl der Exazerbationen war in der Azithromycin-Gruppe signifikant geringer als unter Plazebo (1,5 versus 1,8 pro Patient und Jahr), und die Zeit bis zur ersten Exazerbation war signifikant länger (266 versus 147 Tage). Besonders ausgeprägt war der Effekt von Azithromycin bei Patienten ohne Sauerstofftherapie, in den Gold-Stadien II und III und bei Patienten über 65 Jahre. Diese Ergebnisse sind auf den ersten Blick beeindruckend, allerdings muss man auch potenzielle Nebenwirkungen wie Hörverschlechterung und die Entwicklung von Resistenzen gegen Makrolide berücksichtigen. $\mathrm{Zu}$ prüfen wäre eine dreimal wöchentliche statt tägliche Gabe von Azithromycin.

\section{Beziehung zwischen körperlicher Aktivität und Sterb- lichkeit bei COPD}

Die Arbeitsgruppe aus Großhansdorf publizierte kürzlich eine klare Beziehung zwischen körperlicher Aktivität und Letalität [10]. Eine Kohorte von 170 Patienten mit stabiler COPD wurde über durchschnittlich vier Jahre prospektiv untersucht. Neben Spirometrie, Bodyplethysmografie und 6-Minuten-Gehtest wurde auch die körperliche Aktivität mit dem SenseWear ${ }^{\circledR}$-Gerät gemessen. Das Physical Activity Level (PAL) diente zur Quantifizierung der körperlichen Aktivität. Die Letalität betrug insgesamt $15 \%$. Von den aktiven Personen waren nach 48 Monaten noch alle am Leben, von den sehr inaktiven Patienten jedoch nur noch etwa $65 \%$. Das PAL-Niveau erwies sich als bester Prädiktor zur Abschätzung des 4-Jahres-Überlebens. Diese Daten unterstreichen die Bedeutung körperlicher Bewegung für die Gesundheit der COPD-Patienten. Pneumologen müssen den Lungensport vorantreiben und dürfen nicht mit den 750 Gruppen in Deutschland zufrieden sein, eine Zahl, die im Vergleich zu mehr als 10000 Herzsportgruppen viel zu gering sind. Sport und ambulante Rehabilitation sind neben der medikamentösen Therapie wesentliche Bausteine der Betreuung von COPD-Patienten.

\section{Update der GOLD-Leitlinie \\ $\nabla$}

\section{Michael Pfeifer, Regensburg}

Seit November 2011 steht eine aktualisierte Version der GOLDLeitlinie als Zusammenfassung zur Verfügung, die im Januar 2012 freigegeben wurde.

Die Definition der COPD beinhaltet neuerdings, dass Exazerbationen ebenso wie Komorbiditäten beim individuellen Patienten zum Schweregrad der Erkrankung beitragen. Dagegen wurde der frühere Hinweis auf eine nicht normale Entzündungsreaktion herausgenommen. Den Komorbiditäten ist ein eigenes Kapitel gewidmet. Darin werden auch Depressionen und das Bronchialkarzinom angesprochen.
Bei der medizinischen Versorgung der Patienten wird betont, dass Risikofaktoren reduziert werden sollen. Dazu gehört vor allem der Verzicht auf Zigarettenrauchen und zusätzlich das Verhindern einer Exposition gegenüber anderen schädlichen inhalierbaren Partikeln. Betont wird, dass das FEV1 allein den Verlauf der Erkrankung nur unzureichend beschreiben kann. Neben der Symptomreduktion soll die Behandlung darauf gerichtet sein, Anzahl und Schweregrad der Exazerbationen zu verringern. Therapien, die den langfristigen Verlust an Lungenfunktion wirksam stoppen können, stehen nach Auffassung der Autoren bisher nicht zur Verfügung.

\section{Medikamentöse Therapie}

Langwirksame Bronchodilatatoren (Beta-2-Sympathomimetika und Anticholinergika) sollen gegenüber kurzwirksamen Substanzen den Vorzug erhalten. Patienten mit erhöhtem Risiko für Exazerbationen sollen zusätzlich mit inhalativen Kortikosteroiden behandelt werden. Problematisch gegenüber der Vorversion erscheint, dass hierfür keine deutliche Einschränkung der FEV1 vorliegen muss, sodass im Prinzip eine Exazerbation im Vorjahr genügen würde, diese Kombinationstherapie zu rechtfertigen. Eine Monotherapie mit oralen oder inhalativen Kortikosteroiden wird bei COPD nicht empfohlen. Den Phosphodiesterase-Inhibitor Roflumilast erachtet man als „möglicherweise nützlich“, um bei Patienten mit einer FEV1 unter 50\% des Solls, chronischer Bronchitis und häufigen Verschlechterungen die Zahl der Exazerbationen zu reduzieren.

Die Tabellen mit typischen Medikamenten zur COPD-Behandlung beinhalten überraschenderweise auch Fenoterol, Salbutamol und Terbutalin in Tablettenform bzw. als Sirup. Zur transdermalen Anwendung wird Tulobuterol aufgeführt. Als Kombination von inhalativem Steroid und LABA werden Formoterol/Budesonid und Salmeterol/Fluticason genannt, während die Formoterol/Beclometason-Kombination keine Zulassung für COPD besitzt - diese Empfehlungen sind der Internationalität der Empfehlung geschuldet und lassen sich auf hiesige Verhältnisse nicht übertragen.

\section{Mehrdimensionalität der COPD}

Ein vollkommen neues Element der Leitlinie ist eine 4-Felder-Tafel zur Assoziation zwischen Symptomen, spirometrischer Klassifikation und dem zukünftigen Risiko von Exazerbationen (๑) Abb. 1).

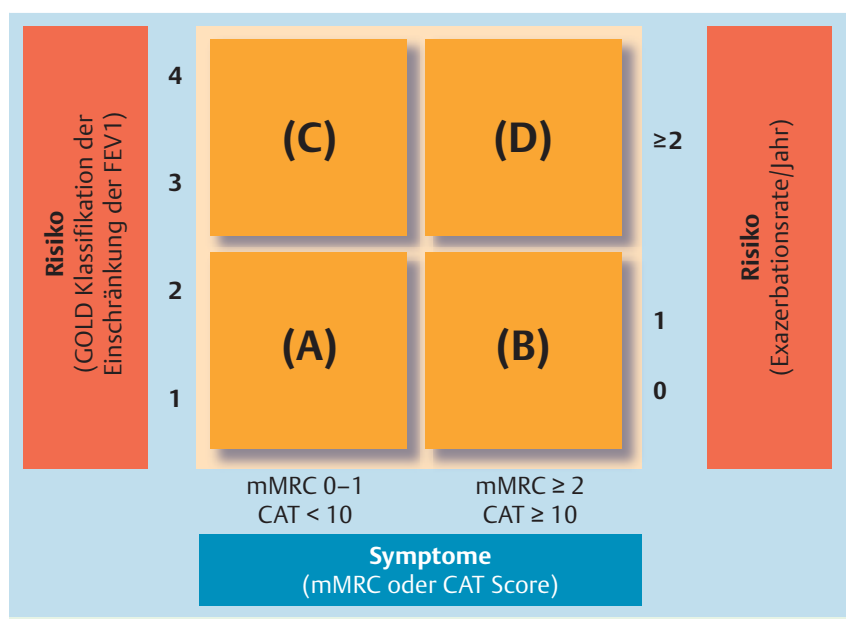

Abb.1 Mehrdimensionale Beurteilung der COPD 
Sie führt in den Zeilen das Ausmaß der Flusslimitation bzw. die Exazerbationen auf. Die Spalten enthalten die Ergebnisse des CAT- oder des mMRC-Fragebogens zur Lebensqualität. Daraus ergeben sich vier Patientengruppen: A: niedriges Risiko, wenig Symptome, B: niedriges Risiko, mehr Symptome, C: hohes Risiko, wenig Symptome und D: hohes Risiko, mehr Symptome. Zur Einordnung wird das jeweils gravierendste Kriterium herangezogen. Die Idee der Mehrdimensionalität ist zwar nachvollziehbar, die Anwendung der 4-Feldertafel erscheint jedoch verwirrend. Offen ist auch die Frage, ob der Patient nach erfolgreicher Therapie in der ursprünglichen Gruppe verbleibt. Eine wissenschaftliche Grundlage für diese neue Einteilung wird nicht genannt; das Schema basiert auf Experten-Erfahrung.

Zur medikamentösen Therapie bei diesen vier Patientengruppen wird unterschieden zwischen Medikamenten erster und zweiter Wahl sowie zwischen alternativen Substanzen. Bei den Bronchodilatatoren werden langwirksame Beta-2-Sympathomimetika und langwirksame Anticholinergika gleichrangig eingeordnet. Für die Nennung von Roflumilast zusätzlich zu einer ICS/Bronchodilatator-Kombination gibt es keine Evidenz, da in den Studien Roflumilast-Patienten mit gleichzeitiger ICS-Therapie nicht untersucht wurden. Ebenfalls erstaunlich ist, dass generell Theophyllin als Alternative genannt wird. Grund dafür könnte sein, dass diese Substanz in der ganzen Welt zu geringen Kosten verfügbar ist. Hier zeigt sich, dass weltweit gültige Leitlinien wie GOLD nicht für jedes Gesundheitssystem sinnvolle Vorschläge machen können. Missverständnisse erscheinen vorprogrammiert, insbesondere bei Allgemeinmedizinern.

Umso wichtiger wird es sein, möglichst bald eine aktualisierte Fassung der deutschen COPD-Leitlinie zu veröffentlichen.

\section{Möglichkeiten und Grenzen der Bildgebung beim Lungenemphysem \\ $\nabla$}

\section{Referent: Hans-Ulrich Kauczor}

Patienten mit chronisch obstruktiver Lungenerkrankung zeigen einen sehr unterschiedlichen Verlauf, der auch eine differenzierte Therapie erfordert. Das CT kann bei der Phänotypisierung der Patienten unterstützen [11]. Die beiden wesentlichen Unterscheidungen sind die zwischen Emphysem-Typ und AtemwegsTyp. Das CT kann dabei als Surrogat-Marker für die histologische Definition des Emphysems dienen, die im Klinikalltag nicht möglich ist.

\section{Emphysem-Typ}

Die histologische Definition des Lungenemphysems beinhaltet verschiedene Subtypen. Das zentrilobuläre Emphysem, der häufigste Typ bei Rauchern, beginnt zentral und schreitet zur Peripherie der Lungenläppchen fort, bis es den gesamten Lobulus erfasst hat. In der Regel findet sich dieser Emphysemtyp vorwiegend in den Oberlappen. Aus einem CT mit $1 \mathrm{~mm}$ Schichtdicke kann der Radiologe optisch das Ausmaß des Emphysems in Größenordnungen von jeweils 25\% einschätzen [12].

Das panlobuläre Emphysem ist gleichmäßiger über die Lunge verteilt, und auch der Lobulus ist gleichförmiger zerstört. Es gilt als typisch für Patienten mit Alpha-1-Antitrypsin-Mangel. Radiologisch findet man eine Aufblähung der Lobuli und eine Rarefizierung der Gefäße.
Eine weitere Form ist das paraseptale Emphysem. Hier sind selektiv subpleurale Lobuli, insbesondere in den Oberlappen vom Emphysem betroffen, und auch entlang der Fissuren dehnt sich die Überblähung aus.

\section{Atemwegs-Typ}

Im Bereich der kleinen Atemwege sind Entzündungsprozesse die wesentliche Ursache für die Obstruktion. Radiologisch findet man bei betroffenen COPD-Patienten typischerweise zentrilobuläre Noduli. Auch Mosaik-Muster, Air trapping und sich verzweigende Verdichtungen sprechen für die Beteiligung der kleinen Atemwege. Entzündungen der großen Atemwege führen zu Symptomen der chronischen Bronchitis. Radiologisch sind sie durch Wandverdickungen, Dilatation der Bronchien und durch die fehlende Verjüngung der Atemwege charakterisiert.

\section{Befundung und Klassifizierung}

Der Radiologe muss entscheiden, ob primär ein Atemwegs- oder ein Emphysem-Typ vorliegt. Er dokumentiert die Beteiligung großer und kleiner Atemwege ebenso wie Art und Ausdehnung des Emphysems. In einer Studie in Heidelberg wurden die Befunde von Teilnehmern eines Lungenkrebs-Screenings mit COPD-Patienten aus der Routineversorgung verglichen. In diesem Kollektiv waren Atemwegs-Typ und Emphysem-Typ ungefähr gleich häufig vertreten. Die Übereinstimmung von drei Befundern für den vorherrschenden Phänotyp war mit 73\% hoch. Auffällig war, dass der Atemwegs-Typ in niedrigen GOLD-Stadien vorherrschte, während der Emphysem-Typ deutlich häufiger bei Patienten in höheren GOLD-Stadien vorkam. Als schwierig erwies sich die Unterscheidung zwischen fortgeschrittenem zentrilobulärem und panlobulärem Emphysem. Das Ausmaß des Emphysems war ein sekundärer Endpunkt in dieser Auswertung, und hier zeigte sich eine zufriedenstellende Übereinstimmung zwischen den drei Befundern.

\section{Densitometrische Auswertung des Emphysems}

Bei guter Bilddatenqualität kann mithilfe spezieller Software das Ausmaß des Emphysems rechnerisch quantifiziert werden. Der Vergleich zwischen dieser densitometrischen und einer visuellen Auswertung durch drei verschiedene Befunder ergab einen Korrelationskoeffizienten von 0,72, wenn die Aufnahmen einen Emphysemanteil zwischen 25 und 50\% aufwiesen. Allerdings waren bei einzelnen Emphysempatienten die Abweichungen zwischen visueller und densitometrischer Befundung erheblich und die 95\% Streubereiche innerhalb von Patientengruppen groß [13].

\section{Verteilung des Emphysems}

Bei der Auswertung der Verteilung des Emphysems bieten sich prinzipiell zwei Möglichkeiten an, nämlich die Einteilung nach Lungenlappen und die nach Lungenhälften (oben vs. unten). Bei Patienten im GOLD-Stadium 0, I oder II ergab sich ein Emphysem-Anteil von insgesamt 9,8\%. Nach Lappen ausgewertet, betrug der Anteil 7,6\%, nach oben/unten 9,6\%, entsprechend einem Unterschied zwischen den Methoden von rund 20\%. In den GOLD-Stadien III und IV, bei Emphysem-Anteilen von 45,1\%, kamen dagegen beide Auswerte-Methoden zu einem sehr ähnlichen Ergebnis.

\section{Atemwegssegmentierung bei zystischer Fibrose}

Bei diesem relativ neuen Verfahren segmentiert die Software zunächst den Tracheobronchialbaum und berechnet eine Zentrallinie entlang eines Bronchus. Der Schnitt senkrecht dazu zeigt 
das bronchiale Lumen und erlaubt zusätzlich die Berechnung der Wanddicke. Die Software mittelt die Messwerte zwischen zwei Verzweigungspunkten des Bronchus.

Bei Patienten mit zystischer Fibrose ergab sich über verschiedene Atemwegs-Generationen ein höherer Gesamtdurchmesser der Atemwege als bei gesunden Kontrollpersonen [14]. Die Messwerte korrelierten gut mit der Spirometrie und dem Atemwegswiderstand, wenn man bei CF die Bronchialgenerationen 5 und 7 betrachtete, während eine solche Assoziation in zentraleren Bronchien nicht nachweisbar war. Hinsichtlich der Fläche des Lumens ergab sich zwischen CF-Patienten und Kontrollpersonen kein wesentlicher Unterschied, abgesehen von der Trachea. Dagegen war die Wanddicke der Bronchien in allen Generationen bei CF signifikant höher.

\section{Bronchuswandfläche bei COPD}

Im COPDGene-Projekt wurde berechnet, wie groß der Anteil der Wandfläche der Bronchien an der Gesamtfläche war [15]. Bei COPD war dieser Anteil größer als bei gesunden Personen, und mit zunehmendem GOLD-Stadium stieg der Anteil der Wandfläche weiter an. Bedauerlicherweise wird in der Literatur nicht immer dezidiert dargestellt, welche Technik verwendet wurde und welche Berechnungen die Software durchgeführt hat. Eine methodische Limitation besteht darin, dass sehr kleine, peripher gelegene Atemwege nur solange darstellbar sind, wie sie größer als ein Pixel sind.

\section{MRT als Alternative zum CT}

In Heidelberg wurden bei Lungenkranken zahlreiche Studien zum Stellenwert der MRT im Vergleich zu klinisch indizierten CT-Untersuchungen durchgeführt [16]. Die Übereinstimmung beider Methoden bei der Darstellung von Emphysem-Typ und -Schweregrad waren nur mäßig [17]. Allerdings ermöglicht das MRT im Unterschied zur CT auch umfassende funktionelle Informationen zu Perfusion und Ventilation. Bei MRT-Untersuchungen mit Kontrastmittel stellte man bei einigen Patienten fest, dass keine komplette Übereinstimmung zwischen emphysematösen Bezirken und fehlender Perfusion besteht [18]. In anderen Fällen erkannte man viele kleine Perfusionsdefekte, ohne dass in denselben Lungenbereichen emphysematöse Veränderungen sichtbar waren.

Ein Perfusions-Imaging liefert auch quantitative regionale Informationen. Man kann darstellen, wie schnell nach der Injektion die Durchblutung bestimmter Lungenbereiche einsetzt [19]. So können Bereiche identifiziert werden, die zwar ausreichend, aber erst verzögert durchblutet werden. Die „Time to peak“ ist hierfür ein geeigneter Messwert.

Bei COPD-Patienten wurden außerdem MRT-Untersuchungen mit hyperpolarisiertem 3-He-Gas durchgeführt [20]. Die Geschwindigkeit, mit der sich die Alveolen füllen, kann damit erfasst werden [21]. Auch die Größe der Alveolen lässt sich darstellen [22]. Denkbar wäre eine Phänotypisierung mit dieser Methode, da der Atemwegs-Typ zu keilförmigen Ventilationsdefekten führt, während beim Emphysem-Typ vor allem die Größe der Alveolen erhöht ist. Eine longitudinale Untersuchung mit dieser Methode ergab bei Ex-Rauchern einen fortschreitenden Funktionsverlust der Lunge, trotz des Rauchstopps [23]. Eine breite Anwendung wird die 3-He-Methode vermutlich nicht finden.

Mit der dynamischen MRT während fortgesetzter Atmung lässt sich hervorragend darstellen, wie stark beim Emphysem die Zwerchfellbeweglichkeit eingeschränkt ist [24].
Derzeit noch in Tierversuchen erprobt wird die neue Methode der Fourier-Dekomposition in der MRT, bei der versucht wird, ohne Kontrastmittel die Durchblutung und die Belüftung der Lunge darzustellen [25]. Diese Methode wird auch beim Menschen anwendbar werden.

\section{Zusammenfassung}

Computertomografische Untersuchungen erlauben eine zuverlässige und hochauflösende Darstellung der Lungenstruktur. Die MRT liefert zusätzlich funktionelle Informationen. Beim Lungenemphysem sollte die visuelle Auswertung der Bildgebung durch den Radiologen mit quantitativen Erfassungsmethoden wie der Densitometrie untermauert werden.

\section{Diskussion}

In der Diskussion wurde die Frage der optimalen Schichtdicke zur Befundung der CT bei COPD erörtert. Im niedergelassenen Bereich werden häufig dickere Schichten als $1 \mathrm{~mm}$ gefahren, wodurch eher 50 als 250 Bilder entstehen. Die wesentlichen Gründe gegen CTs mit vielen dünnen Schichten sind die erforderliche hohe Speicherkapazität und die Rechnerleistung der Computer sowie die vergleichsweise geringere Honorierung der Bildgebung durch die Kostenträger. Dünne Schichten sind erforderlich bei speziellen Fragestellungen, bei Begutachtungen oder auch bei Patienten mit Lungenfibrosen. Eine Indikations-spezifische Anforderung des CTs ist daher sinnvoll. Die Arbeitsgemeinschaft Thoraxdiagnostik der Deutschen Röntgengesellschaft wird hierzu demnächst eine neue Richtlinie erarbeiten.

\section{Erkenntnisse aus COPDGene und ECLIPSE $\nabla$}

\section{Tobias Welte, Hannover}

\section{Genom-Sequenzierung}

Mit der Entwicklung neuer Techniken zur Genom-weiten Analyse der Varianten der Genexpression [26] wurde ein enormer Fortschritt erzielt, der seither vielen Bereichen der Medizin zugute kam. Die riesigen Rechnerkapazitäten, die dafür erforderlich sind, stellen heutzutage kein großes Problem mehr dar. Zur sinnvollen Interpretation der berechneten Daten sind jedoch kompetente Bioinformatiker erforderlich. Medizinern ist es nicht mehr ohne weiteres möglich, das dafür erforderliche Wissen zu kontrollieren und zu überprüfen.

In den letzten Jahren wurden die Sequenzierungs-Techniken fortentwickelt. Die Analysen sind sehr viel schneller und billiger geworden. Während man vor 10 Jahren noch einen Monat benötigte, um 100000 Basenpaare zu analysieren, dauert dies heutzutage nur noch eine Stunde. Parallel dazu sind die Kosten von anfangs mehr als $100000 €$ um den Faktor 1000 gesunken. Ein „Bedside sequencing“ rückt in greifbare Nähe.

Wie hilfreich die gewonnenen Ergebnisse sind, ist noch nicht abschließend zu beurteilen. Normalerweise würde man mithilfe eines Tiermodells versuchen herauszufinden, welche Bedeutung bestimmte genetische Veränderungen haben. Allerdings existieren für die COPD bisher keine etablierten Tiermodelle, sondern nur Annäherungen, wie die ENaC-Knock-out-Maus von Marcus Mall oder Tiermodelle zu den Auswirkungen des Zigarettenrauchs. Ein alternativer Weg besteht darin, Ergebnisse aus Kohortenstudien mit klinischen Daten zu korrelieren und daraus Strategien für die klinische Praxis abzuleiten. Auch dieser Weg hat bisher bei COPD noch keine verwertbaren Ergebnisse geliefert. 


\section{Genetische Varianten beim Asthma bronchiale}

Etwas anders ist die Situation für das Asthma. Hier konnte das von der EU geförderte GABRIEL-Konsortium zeigen, wie genetisch heterogen das Asthma bei Kindern und Erwachsenen ist [27]. Bei Krankheitsbeginn im Kindesalter fand man eine Assoziation mit dem ORMDL3/GSDMB-Locus. Dagegen waren Loci, die stark mit den IgE-Konzentrationen assoziiert waren, nicht mit Asthma assoziiert. Man fand auch keine genetische Ähnlichkeit zwischen Erwachsenen und Kindern mit schwerem Asthma. Dies könnte darauf hinweisen, dass es sich um verschiedene Erkrankungen handelt.

\section{COPDGene ${ }^{\circledR}$-Projekt}

Mit dem COPDGene-Projekt war die Hoffnung verbunden, die Pathophysiologie der COPD besser zu verstehen, die unterschiedlichen klinischen Phänotypen klarer beschreiben zu können, Biomarker für die Diagnostik und Prognose zu identifizieren und die Patienten zielgerichteter medikamentös behandeln zu können. Das Projekt findet in 21 US-amerikanischen Behandlungszentren statt und ist eine der größten Studien zur Untersuchung der genetischen Faktoren, die der COPD zugrunde liegen. Die bisherigen Ergebnisse sind jedoch noch nicht überzeugend.

\section{ECLIPSE-Studie}

Die ECLIPSE-Studie (Evaluation of COPD Longitudinally to Identify Predictive Surrogate End-points) wurde in 46 Zentren in Europa, Nordamerika und Neuseeland durchgeführt. Deutsche Zentren nahmen an dem Projekt bedauerlicherweise nicht teil. ECLIPSE soll die zugrunde liegenden Krankheitsmechanismen besser beschreiben und Biomarker identifizieren, die sich als Surrogatendpunkte für Studien und zur Vorhersage der Krankheitsprogression eignen. Über drei Jahre wurden drei Patientenkohorten mit COPD (GOLD-Stadium II, III und IV) mit rauchenden und nicht rauchenden Kontrollpersonen verglichen [28]. Das Sponsoring dieses Projektes (mehr als 30 Millionen Euro) lag bei GlaxoSmithKline, und Mitarbeiter dieser Firma sind Mitglied des Steering Komitees und des wissenschaftlichen Komitees.

Betrachtet man die ECLIPSE-Patientencharakteristika, so fällt auf, dass das Durchschnittsalter der untersuchten COPD-Patienten bei 63 Jahren lag, mit einer Standardabweichung von 7 Jahren [29]. Wie in den meisten COPD-Therapiestudien wurden daher kaum Aussagen über Patienten generiert, die älter als 75 Jahre sind und eine wesentliche Gruppe der COPD-Patienten ausmachen. Unerwartet waren die Ergebnisse des 6-Minuten-Gehtests in der GOLD-II-Gruppe, die mit durchschnittlich $406 \mathrm{~m}$ relativ schlecht waren; erwarten würde man mehr als $500 \mathrm{~m}$.

\section{Ergebnisse aus ECLIPSE}

Ein wesentliches Ergebnis besteht in der Erkenntnis, dass die COPD eine genetisch und klinisch heterogene Erkrankung ist [30]. Die Ergebnisse der Lungenfunktionsprüfung korrelierten nur mäßig mit dem klinischen Schweregrad der Erkrankung. Dementsprechend kann die Flusslimitation nicht als Anhaltspunkt für die Schwere oder Aktivität der Erkrankung verwendet werden.

In einer anderen Auswertung wurde der Phänotyp des „häufigen Exazerbierers“ beschrieben [29]. Exazerbationen wurden mit zunehmendem COPD-Schweregrad häufiger und schwerer. Betroffene Patienten hatten eine geringere Lebensqualität, und sie wiesen häufiger einen gastroösophagealen Reflux auf. Im Langzeitverlauf traten ausgeprägtere Schädigungen ein, und es könnte sein, dass häufige Exazerbierer eine andere Therapie benötigen als die übrigen Patienten. Der wichtigste Risikofaktor für eine Exazerbation war eine Exazerbation im vorausgegangenen Jahr. Ob die Sputum-Neutrophilie als Biomarker dienen kann, wurde in einer weiteren Auswertung untersucht [31]. Über die verschiedenen GOLD-Stadien gab es eine erhebliche Variation der Neutrophilenzahl im Sputum. Die Zellzahl war weder assoziiert mit dem Verlauf der FEV1 oder dem Ausmaß des Emphysems, sodass sich daraus kein prädiktiver Wert ergab.

Ein gewisser Zusammenhang bestand zwischen der Zahl der Exazerbationen und den Serumkonzentrationen des Surfactant-Proteins D (SP-D) [32]. Im Unterschied zu den Surfactant-Proteinen B und $\mathrm{C}$, die die Oberflächenspannung in den Alveolen beeinflussen, sind die Proteine A und D relevante Komponenten der angeborenen Immunität. Patienten mit COPD hatten in der ECLIPSE-Studie höhere SP-D-Serumspiegel als Kontrollpersonen. Allerdings bestand keine Korrelation zum GOLD-Stadium. Nach einer vierwöchigen Therapie mit Prednisolon nahmen die Konzentrationen von Surfactant-Protein D signifikant ab, sodass SPD als Marker bei der Entwicklung antiinflammatorischer Therapien genutzt werden könnte.

Im Rahmen einer internationalen Zusammenarbeit wurden neben der ECLIPSE-Gruppe auch andere definierte Kohorten herangezogen, um die Empfänglichkeit für die Entwicklung einer COPD mithilfe genetischer Marker zu identifizieren [33]. Nachdem 500000 verschiedene Marker untersucht worden waren, fand sich im FAM13A-Gen auf dem Chromosom 4 ein Locus, der signifikant mit COPD assoziiert war. Die R-Werte lagen allerdings nur bei 0,15 bis 0,25 , das heißt, die Wahrscheinlichkeit für eine COPD ist nur um etwa $20 \%$ größer als ohne diese Variante. Für Einzelpersonen lässt sich aus dieser genetischen Analyse daher keine Aussage treffen. Hier liegt ein Kernproblem der aktuellen genetischen Diagnostik.

Polymorphismen des Surfactant-Proteins D waren ebenfalls mit COPD assoziiert [34]. Auch bei dieser Analyse wurden Patienten aus mehreren internationalen Populationen ausgewertet. Ein bestimmter Polymorphismus war zwar mit spirometrischen Phänotypen assoziiert, jedoch bestanden Unterschiede in den vier untersuchten Studienpopulationen.

Gene, die aus Zellen im induzierten Sputum gewonnen wurden, wurden mit dem spirometrischen und radiologischen Schweregrad der COPD in Beziehung gesetzt [35]. Man konnte Kandidaten-Gene identifizieren, die für die molekulare Pathophysiologie der COPD eine Rolle spielen könnten. Insbesondere die Expression des Interleukin-18-Rezeptors war bei COPD deutlich höher als bei Ex- oder Nichtrauchern (23\% versus 2\%). Die Kernfrage, ob es sich um eine Reaktion oder um eine Ursache handelt, bleibt unbeantwortet, solange die Pathomechanismen nicht abgeklärt sind, beispielsweise durch Versuche an IL-18 R-Knock-out-Mäusen.

\section{Ethische Überlegungen}

Die schnelle Entwicklung der Sequenzierungs-Technologie wird sich in Zukunft fortsetzen, parallel zu einer rasanten Zunahme des bioinformatischen Wissens. Die Sinnhaftigkeit des genetischen Ansatzes hat sich zwar schon in einigen Bereichen gezeigt, bei der COPD bestehen jedoch wegen der sehr inhomogenen Population besondere Schwierigkeiten. Das ethische Dilemma besteht darin, ob wir wirklich alles über uns wissen wollen und wie wir mit dem Wissen leben können. Aus praktischer Sicht stellt sich die Frage, ob eine „personalisierte Medizin“ in der Praxis überhaupt umsetzbar ist, solange es nicht einmal gelingt, Lungenfunktionsuntersuchungen in der Allgemeinmedizin zu 
etablieren. Die diagnostischen Fortschritte müssen sich in sinnvolle Therapien umsetzen lassen, um wirklichen Nutzen zu bringen. Bevor man weiter in großem Rahmen in diese Technologie investiert, sollte man sich der Diskussion um die ethische Dimension stellen.

\section{Biomarker bei der COPD \\ $\nabla$}

\section{Robert Bals, Homburg}

Biomarker sind Indikatoren für normale biologische oder pathologische Prozesse oder auch für das Ansprechen auf pharmakologische Interventionen. Ebenso können sie als Surrogatmarker für klinische Endpunkte dienen. Die Anwendung von Biomarkern umfasst sowohl die Diagnose (Beispiel: Troponin beim akuten Koronarsyndrom) als auch die Therapiesteuerung (Beispiel: HbA1C beim Diabetes mellitus). Beispiele für in der Pneumologie verwendete Biomarker sind der CRB-65-Score, das CRP oder das Copeptin.

Wenn sich ein Patient mit Luftnot vorstellt, wäre es hilfreich, wenn ein Biomarker die Diagnose COPD oder Asthma ermöglichen würde. Wünschenswert wären auch Indikatoren für den zu erwartenden Verlauf, beispielsweise um Patienten mit häufigen Exazerbationen früh identifizieren zu können oder um einen speziellen Phänotyp zu erkennen. Ein spezifischer Biomarker für COPD wurde bisher jedoch noch nicht etabliert. Die Diagnose der COPD wird weiterhin klinisch gestellt.

Eine definierte genetische Risikokonstellation besteht beim Alpha-1-Antitrypsinmangel. Nach Inhalation von Noxen kann dieser Defekt zu einem raschen Abfall der FEV1 beitragen. Dies zeigte sich bei 90 Feuerwehrleuten in New York, die am 11.September 2001 während der Katastrophe am World Trade Center den Brand bekämpften [36]. In den darauf folgenden vier Jahren hatten die 11 Feuerwehrleute mit moderatem oder schwerem Alpha-1-Antitrypsinmangel einen signifikant stärkeren FEV1Verlust als Personen mit normalem AAT-Phänotyp. Bereits die einmalige Exposition induzierte bei diesen Patienten eine deutliche Lungenschädigung.

Prognostische Marker für den Langzeitverlauf der COPD wurden in einer Übersichtsarbeit zusammengestellt [37]. Insgesamt ist die Datenlage jedoch noch wenig ergiebig. Das CRP als klassischer Parameter für systemische Entzündungsprozesse war bei den meisten, jedoch nicht bei allen Rauchern mit COPD erhöht [38]. Das Clara cell secretory protein-16 (CC-16), das in den Clara-Zellen der Lunge produziert wird, wurde bei Patienten in verschiedenen GOLD-Stadien gemessen [39]. Dabei zeigten sich nur minimale Unterschiede zwischen den Gruppen, die weder für die klinische Versorgung noch für Studien relevant waren.

In der ECLIPSE-Studie waren folgende Parameter mit einem verstärkten FEV1-Abfall assoziiert: aktuelles Rauchen, bronchiale Hyperreagibilität und Emphysem [6]. Von den mituntersuchten Biomarkern zeigten nur CRP und CC-16 eine Korrelation mit dem Verlust der FEV1. Dieser betrug jedoch nur $4 \mathrm{ml}$ FEV1 pro Jahr und war damit klinisch nicht relevant.

Eine andere Auswertung der ECLIPSE-Daten von 201 COPD-Patienten bezog sich auf 34 verschiedene Biomarker [40]. Auch hier konnten keine klinisch relevanten Biomarker gefunden werden, die den Verlauf der Erkrankung anzeigen würden. So bleibt die Schlussfolgerung der Übersichtsarbeit von Jones und Agusti aus dem Jahr 2006 [41] weiterhin gültig, dass verlässliche Biomarker für die Versorgung von COPD-Patienten fehlen.
Hier setzt ASCONET/COSYCONET an, das vom Bundesministerium für Bildung und Forschung geförderte Kompetenznetz für Asthma und COPD (http://www.asconet.net). Biomarker sind ein Schwerpunkt dieses Projektes. Von den angestrebten 1500 Patienten wurden zwischen Oktober 2009 und Dezember 2011 bereits 700 Personen rekrutiert.

\section{Messung der Lebensqualität und CAT \\ $\nabla$}

\section{Christian Taube, Leiden}

Arzneimittelzulassungsbehörden fordern zunehmend das Einbeziehen der Lebensqualität und der subjektiv empfundenen Krankheitsauswirkungen, wenn es um die Darstellung der Wirksamkeit neuer Medikamente geht. Zur Messung der Lebensqualität, des Gesundheitsstatus und der Dyspnoe wurden verschiedene Instrumente entwickelt und überprüft.

\section{Dyspnoe}

Die Dyspnoe ist ein Schlüsselsymptom bei COPD. Die Messung dieser als unangenehm empfundenen, erschwerten Atemtätigkeit ist mit verschiedenen Instrumenten möglich. Am bekanntesten ist die Borg-Skala, bei der mit 0 eine nicht wahrnehmbare und mit 10 die maximale Dyspnoe ausgedrückt wird [42]. Für die Darstellung von Veränderungen nach Therapie kann auch eine stufenlose Skala, wie z.B. eine visuelle Analogskala (VAS) verwendet werden. Ein weiteres Instrument ist die Medical Research Council (MRC)-Skala. Diese beschreibt die Dyspnoe in fünf Kategorien und verwendet dazu eine Skala von 0 bis 4 Punkten (keine bis maximale Dyspnoe) [43].

Mit diesen Dyspnoe-Skalen kann der Effekt von Interventionen, wie z.B. Medikamentengabe, auf die Dyspnoe evaluiert werden. Die Borg-Skala ist empfindlich genug, um nach Intervention eine Verbesserung der Dyspnoe anzuzeigen. Dies ließ sich beispielsweise bei Messung der Belastungsdyspnoe nach dreiwöchiger Anwendung kurzwirksamer inhalativer Bronchodilatatoren dokumentieren [44]. Auch bei der Anwendung langwirksamer Bronchodilatatoren konnte eine Reduktion der Dyspnoe unter Belastung mit Hilfe der Borg-Skala gemessen werden [45]. Die Abnahme der Dyspnoe nach Inhalation eines Beta-2-Agonisten, gemessen mit der VAS-Skala, korrelierte nur bedingt mit Änderungen der Lungenfunktion. Dabei scheinen Änderungen der inspiratorischen Lungenfunktionsparameter besser mit der Änderung des subjektiven Befinden zu korrelieren als exspiratorische [46]. Im Unterschied dazu bestand keine Assoziation zwischen VAS und exspiratorischen Parametern wie der FEV1. Auch bei der Verwendung von einfachen inspiratorischen Peak-Flow-Metern ist eine Korrelation zwischen Änderung der Symptomatik und inspiratorischen Parametern nachweisbar [47].

Parameter der Dyspnoe werden auch in sogenannten CompositeScores mitberücksichtigt. Als bekanntestes Beispiel ist der BODEScore zu nennen, der vier verschiedene Parameter, FEV1, 6-Minuten-Gehtest, MRC-Dyspnoe-Skala und Body-Mass-Index, zu einem multidimensionalen System zusammengefasst [48]. Mit 0 Punkten wird der bestmögliche Zustand beschrieben, die maximale Punktzahl beträgt 13. Dieser Score korreliert bei COPD mit der Mortalität. 


\section{Fragebögen zur Lebensqualität}

Bei der Lebensqualität unterscheidet man zwischen der generischen und der krankheitsspezifischen Lebensqualität. Letztere wird bei der COPD am häufigsten mit dem SGRQ gemessen, dem St. George's Respiratory Questionnaire [49]. Er setzt sich zusammen aus den drei Subskalen: Symptome (8 Fragen), Aktivität (16 Fragen) und Auswirkungen (26 Fragen). Für jede Subskala bedeutet 0 das beste Ergebnis und 100 die maximale Beeinträchtigung. Typische Beispiele für nicht krankheitsspezifische Lebensqualität-Instrumente sind der SF (Short Form)-36 und der SF-12.

Der SGRQ korrelierte signifikant mit der körperlichen Belastungsfähigkeit, gemessen mit dem 6-Minuten-Gehtest. Dies galt sowohl für den SGRQ-Gesamt-Score $(r=0,61)$ als auch für den Teilbereich körperliche Aktivität $(r=0,59)$ [50]. Es bestand also ein Zusammenhang zwischen limitierter Aktivität und Lebensqualität. Zusätzlich wurde in dieser Untersuchung deutlich, dass die Lebensqualität erwartungsgemäß eine andere Domäne abbildet als die Lungenfunktion. Aber auch soziokulturelle Faktoren spielen bei der Evaluation von Dyspnoe eine Rolle. In einer Analyse bei niedergelassenen Ärzten unterschieden sich Patienten aus verschiedenen europäischen Ländern in den Mittelwerten der SGRQ-Scores, wobei die günstigsten Ergebnisse in den Niederlanden erhoben wurden [51].

Als minimaler klinisch bedeutsamer Unterschied wird beim SGRQ eine Differenz von 4 Punkten angesehen. Dieses kann durch medikamentöse Intervention durchaus erreicht werden. Als Beispiel sei die UPLIFT-Studie genannt, die unter Behandlung mit einem langwirksamen Anticholinergikum eine Verbesserung des Gesamtscores von 6 Punkten nach sechs Monaten Therapie zeigte, also einen sichtbaren und relevanten Effekt [52]. Auch eine Zunahme der Dyspnoe wird von diesen Fragebögen erfasst. COPD-Patienten mit Exazerbation hatten im Vergleich zum stabilen Zustand [51] signifikant schlechtere Ergebnisse in den Lebensqualitäts-Fragebögen SGRQ, SF-12 und dem FACIT-Score.

\section{Kürzere Fragebögen}

Der SGRQ ist ein umfassender und aufwändiger Fragebogen, dessen Beantwortung viel Zeit und Ressourcen erfordert, wie z.B. einen PC für die Auswertung. Ein Versuch, das Original zu kürzen, um die Durchführung des Tests zu beschleunigen, wurde unter Verwendung der Daten von mehr als 1000 COPD-Patienten unternommen [53]. Der auf 40 Fragen gekürzte Bogen wurde anschließend bei 63 COPD-Patienten in Rehabilitation überprüft. Dabei lieferte der neue SGRQ-C vergleichbare Resultate wie das Original.

\section{COPD Assessment Test, CAT}

Ein erheblich kürzerer Fragebogen wurde erstmals beim Kongress der ERS in Wien im September 2009 vorgestellt [54]. Der COPD Assessment Test (CAT) besteht nur aus 8 Fragen, die ohne großen Aufwand von den Patienten beantwortet werden können. Sie beziehen sich auf Husten, Sputum, Atemnot, Aktivität zuhause und außer Haus, Zuversicht, Schlaf und Energie. Die sechsstufige Skala wird mit 0 bis 5 Punkten (keine bis maximale Probleme) beantwortet, sodass sich ein maximal möglicher Score von 40 ergibt. In der Original-Publikation zeigte sich eine gute Korrelation zwischen CAT und SGRQ. Aber auch im CAT sind regionale Unterschiede nachweisbar. Im Rahmen einer europäischen Untersuchung waren in Belgien die Mittelwerte am höchsten (mittlerer CAT-Score 21,5) und in den Niederlanden am niedrigsten (CAT-Score 16,0).
Auch Verschlechterungen werden mit Hilfe dieses kurzen Fragebogens erfasst. Exazerbationen waren mit einem Anstieg um etwa 5 Punkte verbunden.

In einer aktuellen Publikation erfolgte ein direkter Vergleich zwischen den Ergebnissen von CAT und SGRQ [55]. Dabei wurden vier CAT-Kategorien gebildet, mit denen jeweils eine geringe, mittlere, starke oder sehr starke Beeinträchtigung der Lebensqualität assoziiert war. Die schlechteste Gesundheit wurde ausgedrückt durch die Unfähigkeit, sich vom Bett oder vom Stuhl aus fortzubewegen, hinfällig oder invalide zu sein und keine Hausarbeit mehr machen zu können.

Wie gut der CAT-Score auf Interventionen reagiert, kann noch nicht abschließend beurteilt werden. Nach einem achtwöchigen Reha-Programm verbesserte sich das CAT-Ergebnis zwar im Mittel, jedoch bestand eine große Streuung der Einzelwerte [56].

Insgesamt muss man konstatieren, dass der CAT einen geradezu kometenhaften Aufstieg erfahren hat, wenn er bereits zwei Jahre nach Erstpublikation Eingang in die aktualisierten GOLD-Guidelines erhalten hat. Dies verwundert umso mehr, als es bisher nur wenige Anwendungsstudien zum CAT gibt und weder die Schweregradeinteilung noch die minimale klinisch relevante Differenz festgelegt werden konnte. Experten sind dementsprechend skeptisch, ob die Orientierung der COPD-Therapie am CAT sinnvoll ist. Zusätzlich muss angemerkt werden, dass das Copyright für den Test von einer großen pharmazeutischen Firma gehalten wird. Für jede Anwendung in klinischen Studien muss eine Erlaubnis eingeholt werden. Es wäre wünschenswert, dieses Instrument den Fachgesellschaften, wie z.B. der ERS, zur Verfügung zu stellen.

\section{Asthma-Management 2011 und GINA-Guidelines \\ $\nabla$}

\section{Heinrich Worth, Fürth}

\section{Asthma-Kontrolle}

Die Therapie des Asthmas hat zum Ziel, die Erkrankung zu kontrollieren und Exazerbationen, Verlust von Lungenfunktion oder andere Verschlechterungen zu minimieren. Die GINA-Leitlinien geben ein Stufenschema zur medikamentösen Behandlung vor, das sich ebenfalls am Konzept der Asthmakontrolle orientiert. Dabei spielt die Einschätzung durch den Patienten selbst eine entscheidende Rolle.

Dass die Angaben des Patienten nicht immer zuverlässig zu verwerten sind, zeigt eine eigene aktuelle randomisierte kontrollierte Studie bei 39 Asthmatikern [57]. Die Patienten erhielten entweder eine Behandlung mit Salbutamol, Schein-Akupunktur oder Plazebo, oder es erfolgte keine Intervention. Bei der Messung der FEV1 ergaben sich klare Unterschiede zwischen den Behandlungen: Salbutamol führte zu einem Anstieg um 20\%, während in den anderen Gruppen nur ein 7\%iger Anstieg der FEV1 zu verzeichnen war. Dagegen gaben die Patienten subjektiv in allen drei Behandlungsgruppen eine Verbesserung zwischen $45 \%$ und $50 \%$ an, im Unterschied zur Nicht-Intervention mit nur $21 \%$. Für den behandelnden Arzt ergibt sich aus diesen Daten die Frage, ob man sich wirklich primär nur nach der Einschätzung des Patienten richten kann oder doch Werte der Lungenfunktion benötigt.

\section{Stellenwert von FeNO}

Eine Hilfe für die Therapiesteuerung können Messungen der FeNO-Werte sein, die das Ausmaß der Entzündungsprozesse beschreiben. Die gemessenen Konzentrationen werden allerdings 
durch Faktoren wie Raucherstatus, Energie oder Infektionen maßgeblich beeinflusst [58]. Ob bei der Interpretation der Werte individuelle Korrekturfaktoren herangezogen werden sollten, wurde in einer aktuellen Studie bei 52 Steroid-naiven Personen mit unspezifischen Atemwegssymptomen untersucht [58]. Für die Diagnose eines Asthmas oder eine Abschätzung der SteroidResponse waren Korrekturfaktoren nicht hilfreich.

Bei schwangeren Frauen mit Asthma bronchiale ist die FeNOMessung hilfreich für die Therapiesteuerung [59]. Konzentrationen von 29 bzw. 16 ppb waren die Grenzen für das Steigern bzw. Reduzieren der Steroid-Dosis. Die Patientinnen, deren Behandlung an den FeNO-Werten ausgerichtet wurde, hatten weniger Exazerbationen und geringere Gesamtdosen von inhalativen Kortikosteroiden als die Kontrollgruppe.

\section{LTRA versus ICS und LTRA versus LABA}

Eine aktuelle Publikation beschäftigte sich mit der Frage, ob sich Leukotrien-Rezeptorantagonisten (LTRAs) beim Asthma zur Basistherapie und als Add-on zu inhalativen Steroiden (ICS) eignen [60]. In zwei offenen, „pragmatischen“ Studien bei niedergelassenen Ärzten erhielten suboptimal kontrollierte Patienten mit Asthma über zwei Jahre lang Leukotrien-Antagonisten als Alternative zur üblichen Asthmatherapie: In der Basistherapie-Studie erfolgte ein Vergleich zwischen LTRA und inhalativem Kortikosteroid, und in der Add-on-Studie wurde zusätzlich zum inhalativen Steroid entweder ein Leukotrien-Antagonist oder ein langwirksames Beta-2-Sympathomimetikum (LABA) verabreicht. Die Ergebnisse für Peak flow, Lebensqualität und Asthmakontrolle waren in allen Behandlungsarmen ähnlich. Die Therapie-Adhärenz der Patienten war mit den Leukotrien-Antagonisten besser: in der Basistherapie-Studie 65\% versus $41 \%$ beim ICS, in der Addon-Studie $74 \%$ versus $46 \%$ beim LABA. Untersucht wurde auch, wie viele Patienten den Therapiearm wechseln. Von den Patienten der LTRA-Gruppe wechselten 46\% auf die Kombination ICS plus LABA, während von den Patienten mit inhalativen Kortikosteroiden als Basistherapie nur 2,6\% zum LTRA wechselten. Dies unterstreicht die gute Wirksamkeit der inhalativen Steroide. Bei der Verschreibung der Asthmatherapie sollten in jedem Fall die Präferenzen des Patienten berücksichtigt werden.

\section{Asthma-Phänotypen}

Bei einem Teil der Patienten mit Asthma ist keine vollständige Kontrolle der Erkrankung erreichbar. Gründe dafür sind unterschiedliche Phänotypen, das gleichzeitige Bestehen einer COPD, mangelnde Therapie-Adhärenz des Patienten oder eine Cortison-Resistenz bei Rauchern. Hinzu kommt aber auch, dass manche Patienten keine vollständige Kontrolle anstreben angesichts der dafür erforderlichen aufwändigeren Dauertherapie.

Das Erscheinungsbild des Asthmas, sein Phänotyp, umfasst die Summe aller ererbten und erworbenen Merkmale der Erkrankung. Beim Asthma umfasst dieses ein breites Spektrum. Außerdem beeinflusst eine Vielzahl von Faktoren den Krankheitsverlauf, wie Allergien, Exazerbationen, bleibender Funktionsverlust (Remodelling) und Komorbiditäten.

Das Zytokin-Netzwerk, das für die Pathophysiologie des Asthmas eine wichtige Rolle spielt, kann auf verschiedenen Ebenen medikamentös beeinflusst werden. Zusätzlich zum seit Jahren eingeführten Anti-IgE, Omalizumab, wurden auch Antagonisten von IL-4 und IL-13 entwickelt.

Pitrakinra ist ein inhalativer Antagonist von IL-4/IL-13 und wurde in einer Phase-2b-Studie bei Patienten mit unkontrolliertem Asthma eingesetzt [61]. In der Gesamtgruppe der 534 Studien- teilnehmer wurden keine relevanten Effekte erzielt, jedoch in speziellen Untergruppen von Patienten, nämlich bei eosinophilem Asthma, bei FeNO-Werten im oberen Drittel sowie bei Patienten, die homozygot für ein bestimmtes Allel des IL-4-Rezeptor-Gens waren. Hier ergab sich eine relative Reduktion der Exazerbations-Frequenz um $48 \%$ bis $88 \%$, während die Gesamtgruppe nur eine Abnahme um 29\% aufwies. Die Analyse von Polymorphismen am IL-4-Rezeptor ermöglichte eine Voraussage des Ansprechens auf die Pitrakinra-Therapie.

Ein Anti-IL-13-Antikörper, Lebrikizumab, wurde ebenfalls bei mittelgradig bis schwer erkrankten Patienten mit unkontrolliertem Asthma untersucht [62]. Die Patienten wurden u.a. nach der Konzentration von Periostin im Serum (hoch oder niedrig) separat ausgewertet. IL-13 induziert die Bildung des Signalproteins Periostin in den Bronchialepithelzellen. Bei hohen Periostin-Konzentrationen wurde ein deutlich größerer Anstieg der FEV1 erreicht als bei niedrigen Serumspiegeln (8,2\% versus $1,6 \%$ Verbesserung). Auch hier zeigte sich also je nach Asthma-Phänotyp ein differenziertes Ansprechen auf die Medikation.

Während derzeit das Stufenschema zur Asthmabehandlung einer empirischen Therapie entspricht, könnte die Zukunft darin liegen, Phänotyp-spezifisch für jeden Patienten die individuell beste Medikation zu identifizieren.

\section{Biomarker beim Asthma \\ $\nabla$}

\section{Robert Bals, Homburg}

Für das Asthma wurden bereits mehrere Biomarker identifiziert. Die expiratorische Stickstoffmonoxid-Konzentration (FeNO) hilft bei der Diagnose des Asthmas bzw. einer eosinophilen Atemwegsinflammation und gilt als Indikator für das Ansprechen auf Steroide [63]. Unterteilt man das Asthma in die vier Formen eosinophiles, neutrophiles und pauciimmunes Asthma sowie Asthma mit hohem IgE, so erwartet man ein Ansprechen auf die Therapie mit Omalizumab nur bei Patienten mit hohem IgE.

Interleukin-13 wird von Th2-Helferzellen produziert und induziert in Bronchialepithelzellen die Produktion von Periostin. Da Periostin beim Asthma zur Fibroblasten-Akkumulation und damit zum Remodeling der Atemwege beiträgt [64], kann es als Surrogatparameter für die IL-13-Aktivität genutzt werden. Tatsächlich verbesserte ein Anti-Interleukin-13-Antikörper, Lebrikizumab, die Lungenfunktion vor allem bei Patienten mit hohen Periostin-Konzentrationen im Serum [62].

In dem Maße, wie die Pathomechanismen des Asthmas besser verstanden werden, können Biomarker helfen, bestimmte Medikamente gezielter einzusetzen. So profitieren Patienten mit eosinophilem Asthma und solche mit hohem IgE von Steroiden, Omalizumab und Lebrikizumab, während man ein gutes Ansprechen auf Tiotropium oder Rofluminast vor allem bei neutrophilem oder pauciimmunem Asthma erwarten könnte. Zulassungsbehörden wie die FDA haben für klinische Prüfungen klare Anforderungen an primäre Endpunkte formuliert, einschließlich der Angabe der kleinsten klinisch relevanten Differenz.

Der Einsatz von Biomarkern in Arzneimittelstudien bei COPD stößt derzeit noch auf diverse Schwierigkeiten. 


\section{Anticholinergika in der Therapie des Asthma bronchiale \\ $\nabla$}

\section{Winfried J. Randerath, Solingen}

Im Bronchialsystem sind die meisten parasympathischen Fasern afferent, also sensorisch. Für die nächtliche Obstruktion ist vor allem die Reflex-Bronchokonstriktion wichtig, die auf zirkadianen Tonusschwankungen beruht. Von Anticholinergika ist bekannt, dass sie diese Reflex-Bronchokonstriktion hemmen, während Histamin oder Leukotriene darauf keinen Einfluss haben.

\section{Akuter Asthmaanfall und Metacholinprovokation}

In einer Meta-Analyse wurden Studien mit insgesamt 3600 Kindern und Erwachsenen zusammengefasst, die beim akuten Asthma entweder Betamimetika oder eine Kombination aus Betamimetikum und Anticholinergikum erhielten [65]. Die zusammenfassende Auswertung ergab einen signifikanten Vorteil durch das Anticholinergikum als Add-on-Therapie, während in den einzelnen Studien meist kein signifikanter Effekt erzielt worden war.

In einer kleineren doppelblinden, plazebokontrollierten Studie erhielten 12 Patienten mit extrinsischem Asthma MetacholinProvokationen [66]. Geprüft wurden die Auswirkungen einer Tiotropium-Therapie $(10 \mu \mathrm{g}, 40 \mu \mathrm{g}$ oder $80 \mu \mathrm{g})$ im Vergleich zu Plazebo. Der Effekt auf die Bronchodilatation war zwar insgesamt gering. Die bronchiale Hyperreagibilität konnte jedoch durch Tiotropium günstig beeinflusst werden, und zwar mit einem Dosis-abhängigen Effekt.

\section{Tierversuche zum chronischen Asthma}

Dem chronischen Asthma liegen langfristige Entzündungsprozesse zugrunde, an denen auch das cholinerge System beteiligt ist [67]. Epithel- und Entzündungszellen bilden muskarinerge Rezeptoren, Acetylcholin wird produziert, und der Vagotonus ist erhöht. Beim Atemwegs-Remodeling wird die Proliferation der glatten Muskulatur durch Stimulation des $\mu 3$-Rezeptors gesteigert. Umgekehrt konnten im Tierversuch die Zunahme der Muskelmasse und die Umbauprozesse in den kleinen Atemwegen durch eine Behandlung mit Tiotropium reduziert werden [68]. In einer anderen Studie wurden sensibilisierte Mäuse akut und chronisch mit Ovalbumin belastet [69]. Die Behandlung mit Tiotropium reduzierte die Th2-Zytokinproduktion und die Atemwegsinflammation und könnte auf diese Weise ein Remodeling begrenzen.

\section{Klinische Studien mit Tiotropium}

In einer Übersichtsarbeit zu Anticholinergika beim Asthma wurde festgestellt, dass Patienten mit nächtlichem Asthma oder mit einem lange bestehenden intrinsischen Asthma besonders gut auf diese Medikamente ansprechen [70].

Auch genetische Determinanten beeinflussen die Wirksamkeit von Tiotropium. In einer prospektiven Studie erhielten $138 \mathrm{~Pa}-$ tienten mit schwerem Asthma über 12 Wochen täglich $18 \mu \mathrm{g}$ Tiotropium [71]. Patienten, die einen Anstieg der FEV1 von mindestens $15 \%$ oder $200 \mathrm{ml}$ gegenüber dem Ausgangswert zeigten, wurden als Responder definiert ( $n=46$ bzw. 33\%). Zusätzlich wurden in dieser Studie genetische Polymorphismen (SNPs) analysiert. Eine logistische Regressionsanalyse zeigte, dass Arg16Gly in ADRB2 (Beta2-Rezeptor-Gen) signifikant mit einem guten Ansprechen auf Tiotropium assoziiert war.
Bestimmte Asthmatiker, nämlich solche mit dem B16-Arg/ArgGenotyp, sprechen nicht immer gut auf langwirksame Betamimetika an. Eine aktuelle klinische Prüfung an 388 Patienten mit diesem Polymorphismus verglich Tiotropium, Salmeterol und Plazebo bei Patienten, die unter hohen Budesonid-Dosen nicht ausreichend kontrolliert waren [72]. Alle Studienteilnehmer inhalierten zunächst vier Wochen lang mit Salmeterol, bevor sie randomisiert wurden. Während der 16-wöchigen Behandlung waren die Peak-flow-Werte unter Tiotropium und Salmeterol deutlich besser als unter Plazebo. Zwischen den beiden Bronchodilatatoren zeigte sich kein signifikanter Unterschied der Wirksamkeit.

Tiotropium wurde auch bei 17 Patienten mit schwerem persistierenden Asthma eingesetzt, von denen 23\% orales Prednisolon einnahmen und 35\% ihre langwirksame Betamimetika wegen Nebenwirkungen oder wegen mangelnder Wirksamkeit wieder abgesetzt hatten [73]. Im induzierten Sputum wurde der relative Anteil von eosinophilen und von neutrophilen Granulozyten analysiert. Nach vier Wochen Tiotropium ergab sich für die Gesamtgruppe ein signifikanter Anstieg der FEV1. Die quantitativ größten Verbesserungen fand man bei Patienten mit einem relativ hohen Anteil von neutrophilen Granulozyten.

In einer doppelblinden, plazebokontrollierten Studie wurde Tiotropium bei 472 Patienten geprüft, bei denen gleichzeitig eine COPD und ein Asthma diagnostiziert worden war [74]. Die Patienten waren länger als ein Jahr mit inhalativen Steroiden behandelt und umfassend vortherapiert worden. Die Wirkung von Tiotropium war deutlich stärker als die von Plazebo, bezogen auf FEV1 und FVC am Morgen sowie auf die symptombezogene Anwendung von Salbutamol.

Auch in einer aktuellen klinischen Prüfung erwies sich Tiotropium bei Patienten mit unkontrolliertem Asthma als wirksam [75]. In die doppelblinde Studie wurden Patienten aufgenommen, die trotz hochdosierter inhalativer Kortikosteroide und langwirksamer Betamimetika keine Asthmakontrolle erreicht hatten. Die Studienteilnehmer inhalierten aus dem Respimat täglich entweder $5 \mu$ g oder $10 \mu \mathrm{g}$ Tiotropium oder Plazebo. Sowohl innerhalb der ersten Stunden nach Anwendung als auch über 24 Stunden erwies sich Tiotropium als deutlich wirksamer als Plazebo, bezogen auf den Anstieg von FEV1 und FVC.

Tiotropium und Salmeterol wurden in einer doppelblinden, 3fach Dummy- und Cross-over-Studie mit der Verdoppelung der ICS-Dosis verglichen [76]. Das Asthma der Patienten war unter inhalativen Steroiden mangelhaft kontrolliert gewesen, und ein positiver Bronchodilatatoreffekt war ein Einschlusskriterium für die Teilnahme. In der Studie erhielt jeder Patient jede Prüfsubstanz. Über jeweils 14 Wochen Therapie waren Tiotropium und Salmeterol signifikant besser wirksam als die Verdopplung der inhalativen Kortikosteroide, bezogen auf Morgen- und AbendPeak-flow-Werte, präbronchodilatatorische FEV1 und die Zahl der Tage mit guter Asthmakontrolle. Statistisch konnte die Nicht-Unterlegenheit von Tiotropium gegenüber Salmeterol gezeigt werden. Erstaunlich war der hohe durchschnittliche BMIWert der Patienten $\left(31 \mathrm{~kg} / \mathrm{m}^{2}\right)$, der auf einen hohen Anteil adipöser Patienten hinweist.

\section{Fazit}

Zu Tiotropium bei Patienten mit Asthma bronchiale gibt es bisher nur wenige Studien. Das Ansprechen dieser Patientengruppe ist heterogen, und in der Regel ist die Wirksamkeit der Betamimetika größer als die der Anticholinergika. Ein gutes Ansprechen darf erwartet werden bei Patienten mit nächtlichem Asthma, 
mit neutrophilem Asthma, bei chronischen Virusinfektionen, bestimmten genetische Subtypen sowie bei Asthma mit koexistierender COPD.

\section{Zusammenfassung \\ $\nabla$ \\ Joachim Lorenz, Lüdenscheid}

Die während der Tagung präsentierten wissenschaftlichen Ergebnisse zur Pathogenese und Klinik der chronisch obstruktiven Lungenerkrankung (COPD) und des Asthma dokumentieren die aktuelle Entwicklungsstufe der Forschung. Das Nebeneinander von Grundlagenarbeit isolierter Forschergruppen einerseits und klinischen Studien andererseits wird zunehmend verlassen. Die Unterstützung durch öffentliche und private Förderung ermöglicht systematische Analysen von wohldefinierten und intensiv untersuchten Patientenkohorten. Beispiele dafür sind die ECLIPSE-Kohorte in den USA und das COSYCONet in Deutschland. Sie dienen dem Ziel, die Ergebnisse aus beiden Forschungsfeldern zusammenzubringen, um sowohl auf der Ebene der klinischen Phänomenologie wie in der Biologie von Asthma und COPD Ansätze für neue Instrumente in der Risikoabschätzung, Diagnostik und Therapie zu finden. Im Jahr 2011 trugen diese Ansätze erste Früchte. Sie werden in der hier vorgelegten Übersicht dargestellt. Die Beiträge über zentrale Publikationen zu COPD und Asthma des Jahres 2011 und über die Neufassung der GOLD-Leitlinie belegen den raschen wissenschaftlichen Fortschritt. Viele bisherige Vorstellungen werden durch neue Daten infrage gestellt. So wird zum Beispiel das pathogenetische Verhältnis von Parenchymdestruktion und bronchialer Obstruktion bei der COPD neu gesehen; die Bedeutung der kardiovaskulären Komorbidität wird neu zu bewerten sein und - damit in Zusammenhang - die Rolle von Betarezeptor-Antagonisten in der Therapie der COPD, ebenso die Rolle von Anticholinergika in der Asthmatherapie. Das GOLD-Komitee unternimmt den Versuch, die multiplen Facetten der COPD in die Form einer praktikablen Klassifikation zu fassen. Die Beiträge über neue Untersuchungen zur Morphologie, Biomarker, Lebensqualität und COPDGene und die ECLIPSE-Kohorte vertieften das Thema der Tagung: Neue Targets in Forschung und Klinik.

\section{Interessenkonflikt}

$\nabla$

R. Bals erhielt Erstattungen von Reisekosten oder Teilnahmegebühren für einen Kongress oder eine Fortbildungsveranstaltung, Honorare für Vorträge, Unterstützung für ein Forschungsvorhaben oder finanzielle Zuwendungen für die Teilnahme an einer Studie von den Firmen Mundipharma, Novartis und Grifols.

H.-U. Kauczor erhielt Honorare für Vorträge sowie für Beratertätigkeit von Siemens und Boehringer Ingelheim.

J. Lorenz erhielt Erstattungen von Reisekosten oder Teilnahmegebühren für Kongresse oder Fortbildungsveranstaltungen sowie Honorare für Vorträge.

M. Pfeifer erhielt Erstattungen von Reisekosten oder Teilnahmegebühren für Kongresse oder Fortbildungsveranstaltungen, Honorare für Vorträge und Unterstützung für ein Forschungsvorhaben oder finanzielle Zuwendungen für die Teilnahme an einer Studie.

W. Randerath erhielt Erstattungen von Reisekosten oder Teilnahmegebühren für Kongresse oder Fortbildungsveranstaltungen, Honorare für Vorträge, Unterstützung für ein Forschungsvorha- ben oder finanzielle Zuwendungen für die Teilnahme an einer Studie sowie Honorar für Beratertätigkeit.

C. Taube erhielt Erstattungen von Reisekosten oder Teilnahmegebühren für Kongresse oder Fortbildungsveranstaltungen, Honorare für Vorträge und für Beratertätigkeit.

Helmut Teschler erhielt Erstattungen von Reisekosten oder Teilnahmegebühren für Kongresse oder Fortbildungsveranstaltungen sowie Honorar für einen Vortrag.

T. Welte erhielt Erstattungen von Reisekosten oder Teilnahmegebühren für Kongresse oder Fortbildungsveranstaltungen, Honorare für Vorträge, Unterstützung für ein Forschungsvorhaben oder finanzielle Zuwendung für die Teilnahme an einer Studie, Finanzierung von Mitarbeitern sowie Honorar für Beratertätigkeit.

H. Worth erhielt Erstattungen von Reisekosten oder Teilnahmegebühren für Kongresse oder Fortbildungsveranstaltungen, Honorare für Vorträge, Unterstützung für ein Forschungsvorhaben oder finanzielle Zuwendung für die Teilnahme an einer Studie sowie Honorar für Beratertätigkeit.

G. Steinkamp erhielt Erstattungen von Reisekosten oder Teilnahmegebühren für Kongresse oder Fortbildungsveranstaltungen sowie Honorare für Medical Writing der Firmen Boehringer Ingelheim, Chiesi, Glaxo Smith Kline, Grifols, Novartis, TEVA und Roche.

\section{Institute}

${ }^{1}$ Klinik für Pneumologie und Internistische Intensivmedizin, Klinikum Lüdenscheid

2 Pneumologie, Allergologie, Beatmungsmedizin, Universitätsklinikum des Saarlandes

${ }^{3}$ Diagnostische und Interventionelle Radiologie, Universitätsklinikum

Heidelberg

${ }^{4}$ Klinik Donaustauf, Universität Regensburg

${ }^{5}$ Klinik für Pneumologie und Allergologie, Krankenhaus Bethanien, Solingen

${ }^{6}$ Medizinisch-wissenschaftliches Publizieren, Schwerin

7 Department of Pulmonology, Leiden University Medical Center

${ }^{8}$ Ruhrlandklinik, Westdeutsches Lungenzentrum, Universitätsklinikum Essen

${ }^{9}$ Klinik für Pneumologie, Medizinische Hochschule Hannover

${ }^{10}$ Medizinische Klinik I, Klinikum Fürth

\section{Literatur}

1 Gershon AS, Warner L, Cascagnette $P$ et al. Lifetime risk of developing chronic obstructive pulmonary disease: a longitudinal population study. Lancet 2011; 378: 991 -996

2 McDonough JE, Yuan R, Suzuki M et al. Small-airway obstruction and emphysema in chronic obstructive pulmonary disease. N Engl J Med 2011; 365: 1567-1575

3 Mitzner $W$. Emphysema-a disease of small airways or lung parenchyma? N Engl J Med 2011; 365: 1637 - 1639

4 Kessler R, Partridge MR, Miravitlles $M$ et al. Symptom variability in patients with severe COPD: a pan-European cross-sectional study. Eur Respir J 2011; 37: 264-272

5 Chang CL, Robinson SC, Mills GD et al. Biochemical markers of cardiac dysfunction predict mortality in acute exacerbations of COPD. Thorax 2011; 66: 764-768

6 Vestbo J, Edwards LD, Scanlon PD et al. Changes in forced expiratory volume in 1 second over time in COPD. N Engl J Med 2011; 365: 11841192

7 Short PM, Lipworth SI, Elder DH et al. Effect of beta blockers in treatment of chronic obstructive pulmonary disease: a retrospective cohort study. BMJ 2011; 342: d2549

8 Bateman ED, Rabe KF, Calverley PM et al. Roflumilast with long-acting beta2-agonists for COPD: influence of exacerbation history. Eur Respir J 2011; 38: $553-560$

9 Albert RK, Connett J, Bailey WC et al. Azithromycin for prevention of exacerbations of COPD. N Engl J Med 2011; 365: 689-698 
10 Waschki B, Kirsten A, Holz $O$ et al. Physical activity is the strongest predictor of all-cause mortality in patients with COPD: a prospective cohort study. Chest 2011; 140: 331 - 342

11 Coxson HO, Mayo J, Lam S et al. New and current clinical imaging techniques to study chronic obstructive pulmonary disease. Am J Respir Crit Care Med 2009; 180: 588-597

12 Kauczor HU, Wielputz MO, Owsijewitsch $M$ et al. Computed tomographic imaging of the airways in COPD and asthma. J Thorac Imaging 2011; 26: $290-300$

13 Owsijewitsch M, Grenier P, Crapo JD. Radiological Society of North America (RSNA). et al. COPD Phenotyping in CT: Comparison of Visual and Densitometric Emphysema Extent Assessment. 2009: SSC04SSC07

14 Quantitative Airway Analysis on Thin-Section MDCT in Cystic Fibrosis - Correlation with Pulmonary Function Testing. 29. 11. 2011

15 Han MK, Kazerooni EA, Lynch DA et al. Chronic obstructive pulmonary disease exacerbations in the COPDGene study: associated radiologic phenotypes. Radiology 2011; 261: 274-282

16 Ley-Zaporozhan J, Puderbach M, Kauczor HU. MR for the evaluation of obstructive pulmonary disease. Magn Reson Imaging Clin N Am 2008; 16: $291-308$, ix

17 Ley-Zaporozhan J, Ley S, Eberhardt R et al. Visualization of morphological parenchymal changes in emphysema: comparison of different MRI sequences to 3D-HRCT. Eur J Radiol 2010; 73: 43-49

18 Ley-Zaporozhan J, van Beek EJ. Imaging phenotypes of chronic obstructive pulmonary disease. J Magn Reson Imaging 2010; 32: 1340 1352

19 Risse F, Eichinger M, Kauczor HU et al. Improved visualization of delayed perfusion in lung MRI. Eur J Radiol 2011; 77: 105-110

20 van Beek EJ, Dahmen AM, Stavngaard T et al. Hyperpolarised 3He MRI versus HRCT in COPD and normal volunteers: PHIL trial. Eur Respir J 2009; 34: $1311-1321$

21 Lehmann F, Knitz F, Weiler $N$ et al. [A software tool for analysis and quantification of regional pulmonary ventilation using dynamic hyperpolarised-(3)He-MRI] Quantitative Untersuchung der Lungenventilation mittels dynamischer MRT von hochpolarisiertem Helium-3. Fortschr Röntgenstr 2004; 176: 1399-1408

22 Woods JC, Choong CK, Yablonskiy DA et al. Hyperpolarized 3He diffusion MRI and histology in pulmonary emphysema. Magn Reson Med 2006; 56: $1293-1300$

23 Kirby M, Mathew L, Wheatley A et al. Chronic obstructive pulmonary disease: longitudinal hyperpolarized (3)He MR imaging. Radiology 2010; 256: $280-289$

24 Tetzlaff $R$, Eichinger M. Magnetresonanztomographie der Atembewegung und Lungenfunktion. Radiologe 2009; 49: 712 - 719

25 Bauman $G$, Lutzen $U$, Ullrich $M$ et al. Pulmonary functional imaging: qualitative comparison of Fourier decomposition MR imaging with SPECT/CT in porcine lung. Radiology 2011; 260: 551 - 559

26 Morley M, Molony CM, Weber TM et al. Genetic analysis of genomewide variation in human gene expression. Nature 2004; 430: 743 - 747

27 Moffatt MF, Gut IG, Demenais F et al. A large-scale, consortium-based genomewide association study of asthma. N Engl J Med 2010; 363: $1211-1221$

28 Vestbo J, Anderson W, Coxson HO et al. Evaluation of COPD Longitudinally to Identify Predictive Surrogate End-points (ECLIPSE). Eur Respir J 2008; 31: 869-873

29 Hurst JR, Vestbo J, Anzueto A et al. Susceptibility to exacerbation in chronic obstructive pulmonary disease. N Engl J Med 2010; 363: $1128-1138$

30 Agusti A, Calverley PM, Celli B et al. Characterisation of COPD heterogeneity in the ECLIPSE cohort. Respir Res 2010; 11: 122

31 Singh D, Edwards L, Tal-Singer $R$ et al. Sputum neutrophils as a biomarker in COPD: findings from the ECLIPSE study. Respir Res 2010; 11: 77

32 Lomas DA, Silverman EK, Edwards $L D$ et al. Serum surfactant protein D is steroid sensitive and associated with exacerbations of COPD. Eur Respir J 2009; 34: 95 - 102

33 Cho MH, Boutaoui N, Klanderman BJ et al. Variants in FAM13A are associated with chronic obstructive pulmonary disease. Nat Genet 2010 . 42: $200-202$

34 Foreman MG, Kong X, DeMeo DL et al. Polymorphisms in surfactant protein-D are associated with chronic obstructive pulmonary disease. Am J Respir Cell Mol Biol 2011; 44: 316 - 322

35 Singh D, Fox SM, Tal-Singer $R$ et al. Induced sputum genes associated with spirometric and radiological disease severity in COPD ex-smokers. Thorax 2011; 66: 489-495
36 Banauch GI, Brantly M, Izbicki G et al. Accelerated spirometric decline in New York City firefighters with alpha-antitrypsin deficiency. Chest 2010; 138: 1116-1124

37 Sin $D D$, Vestbo J. Biomarkers in chronic obstructive pulmonary disease. Proc Am Thorac Soc 2009; 6: 543-545

38 de Torres JP, Cote CG, Lopez MV et al. Sex differences in mortality in patients with COPD. Eur Respir J 2009; 33: 528 - 535

39 Lomas DA, Silverman EK, Edwards LD et al. Evaluation of serum CC-16 as a biomarker for COPD in the ECLIPSE cohort. Thorax 2008; 63: 1058 1063

40 Dickens JA, Miller BE, Edwards LD et al. COPD association and repeatability of blood biomarkers in the ECLIPSE cohort. Respir Res 2011; 12: 146

41 Jones PW, Agusti AG. Outcomes and markers in the assessment of chronic obstructive pulmonary disease. Eur Respir J 2006; 27: 822 832

42 Borg GA. Perceived exertion. Exerc Sport Sci Rev 1974; 2: 131 - 153

43 Bestall JC, Paul EA, Garrod $R$ et al. Usefulness of the Medical Research Council (MRC) dyspnoea scale as a measure of disability in patients with chronic obstructive pulmonary disease. Thorax 1999; 54: 581 586

44 O'Donnell DE, Lam M, Webb KA. Measurement of symptoms, lung hyperinflation, and endurance during exercise in chronic obstructive pulmonary disease. Am J Respir Crit Care Med 1998; 158: 1557-1565

45 O'Donnell DE, Fluge T, Gerken $F$ et al. Effects of tiotropium on lung hyperinflation, dyspnoea and exercise tolerance in COPD. Eur Respir J 2004: 23: $832-840$

46 Taube C, Lehnigk B, Paasch K et al. Factor analysis of changes in dyspnea and lung function parameters after bronchodilation in chronic obstructive pulmonary disease. Am J Respir Crit Care Med 2000; 162 $216-220$

47 Taube C, Rydzy L, Eich A et al. Use of a portable device to record maximum inspiratory flow in relation to dyspnoea in patients with COPD. Respir Med 2011; 105: 316-321

48 Celli BR, Cote CG, Marin JM et al. The body-mass index, airflow obstruction, dyspnea, and exercise capacity index in chronic obstructive pulmonary disease. N Engl J Med 2004; 350: 1005-1012

49 Jones PW, Quirk FH, Baveystock CM. The St George's Respiratory Questionnaire. Respir Med 1991; 85: 25 - 31

50 Jones PW. Activity limitation and quality of life in COPD. COPD 2007; 4 $273-278$

51 Jones $P W$, Brusselle G, Dal Negro RW et al. Health-related quality of life in patients by COPD severity within primary care in Europe. Respir Med 2011; 105: $57-66$

52 Tashkin DP, Celli B, Senn S et al. A 4-year trial of tiotropium in chronic obstructive pulmonary disease. N Engl J Med 2008; 359: 1543 - 1554

53 Meguro M, Barley EA, Spencer S et al. Development and Validation of an Improved, COPD-Specific Version of the St. George Respiratory Questionnaire. Chest 2007; 132: 456-463

54 Jones PW, Harding G, Berry P et al. Development and first validation of the COPD Assessment Test. Eur Respir J 2009; 34: 648 - 654

55 Jones $P W$, Tabberer $M$, Chen $W H$. Creating scenarios of the impact of COPD and their relationship to COPD Assessment Test (CAT) scores. BMC Pulm Med 2011; 11: 42

56 Dodd JW, Hogg L, Nolan J et al. The COPD assessment test (CAT): response to pulmonary rehabilitation. A multicentre, prospective study. Thorax 2011; 66: 425-429

57 Wechsler ME, Kelley JM, Boyd IO et al. Active albuterol or placebo, sham acupuncture, or no intervention in asthma. N Engl J Med 2011; 365 : $119-126$

58 Dressel $H$, de la Motte D, Reichert J et al. Exhaled nitric oxide: independent effects of atopy, smoking, respiratory tract infection, gender and height. Respir Med 2008; 102: 962 -969

59 Powell H, Murphy VE, Taylor DR et al. Management of asthma in pregnancy guided by measurement of fraction of exhaled nitric oxide: a double-blind, randomised controlled trial. Lancet 2011; 378: 983 - 990

60 Price D, Musgrave SD, Shepstone L et al. Leukotriene antagonists as firstline or add-on asthma-controller therapy. N Engl J Med 2011; 364: $1695-1707$

61 Otulana BA, Wenzel SE, Ind PW et al. A Phase 2b Study Of Inhaled Pitrakinra, An Il-4/il-13 Antagonist, Successfully Identified Responder Subpopulations Of Patients With Uncontrolled Asthma. American Journal of Respiratory and Critical Care Medicine 2011; 183: A6179

62 Corren J, Lemanske RF, Hanania NA et al. Lebrikizumab treatment in adults with asthma. N Engl J Med 2011; 365: 1088-1098 
63 Dweik RA, Boggs PB, Erzurum SC et al. An official ATS clinical practice guideline: interpretation of exhaled nitric oxide levels (FENO) for clinical applications. Am J Respir Crit Care Med 2011; 184: 602 - 615

64 Takayama G, Arima K, Kanaji T et al. Periostin: a novel component of subepithelial fibrosis of bronchial asthma downstream of IL-4 and IL13 signals. J Allergy Clin Immunol 2006; 118: 98 - 104

65 Rodrigo GJ, Castro-Rodriguez JA. Anticholinergics in the treatment of children and adults with acute asthma: a systematic review with meta-analysis. Thorax 2005; 60: 740 - 746

66 O'Connor BJ, Towse LJ, Barnes PJ. Prolonged effect of tiotropium bromide on methacholine-induced bronchoconstriction in asthma. Am J Respir Crit Care Med 1996; 154: 876-880

67 Gosens R, Zaagsma J, Meurs $H$ et al. Muscarinic receptor signaling in the pathophysiology of asthma and COPD. Respir Res 2006; 7: 73

68 Gosens $R$, Bos IS, Zaagsma $J$ et al. Protective effects of tiotropium bromide in the progression of airway smooth muscle remodeling. Am J Respir Crit Care Med 2005; 171: 1096-1102

69 Ohta S, Oda N, Yokoe T et al. Effect of tiotropium bromide on airway inflammation and remodelling in a mouse model of asthma. Clin Exp Allergy 2010; 40: $1266-1275$

70 Kanazawa H. Anticholinergic agents in asthma: chronic bronchodilator therapy, relief of acute severe asthma, reduction of chronic viral in- flammation and prevention of airway remodeling. Curr Opin Pulm Med 2006; 12: 60-67

71 Park HW, Yang MS, Park CS et al. Additive role of tiotropium in severe asthmatics and Arg16Gly in ADRB2 as a potential marker to predict response. Allergy 2009; 64: 778 - 783

72 Bateman ED, Kornmann O, Schmidt $P$ et al. Tiotropium is noninferior to salmeterol in maintaining improved lung function in B16-Arg/Arg patients with asthma. J Allergy Clin Immunol 2011; 128: 315-322

73 Iwamoto $H$, Yokoyama A, Shiota $N$ et al. Tiotropium bromide is effective for severe asthma with noneosinophilic phenotype. Eur Respir J 2008; 31: $1379-1380$

74 Magnussen H, Bugnas B, van Noord J et al. Improvements with tiotropium in COPD patients with concomitant asthma. Respir Med 2008; 102 : $50-56$

75 Kerstjens HA, Disse B, Schroder-Babo W et al. Tiotropium improves lung function in patients with severe uncontrolled asthma: a randomized controlled trial. J Allergy Clin Immunol 2011; 128: 308 - 314

76 Peters SP, Kunselman SJ, Icitovic $N$ et al. Tiotropium bromide step-up therapy for adults with uncontrolled asthma. N Engl J Med 2010; 363: $1715-1726$ 